\title{
Metabolic response of the Siberian frog Rana amurensis to anoxia
}

\author{
Sergei V. Shekhovtsov \\ ICG SB RAS, Novosibirsk, Russia \\ shekhovtsov@bionet.nsc.ru
}

\author{
Nina A. Bulakhova \\ IBPN FEB RAS, Magadan, Russia \\ sigma44@mail.ru \\ Ekaterina A. Zelentsova \\ ITC SB RAS, Novosibirsk, Russia \\ zelentsova.ekaterina@gmail.com
}

\author{
Yuri P. Tsentalovich \\ ITC SB RAS, Novosibirsk, Russia \\ yura.tsentalovich@tomo.nsc.ru \\ Daniil I. Berman \\ IBPN FEB RAS, Magadan, Russia \\ dberman@mail.ru
}

\begin{abstract}
The Siberian frog Rana amurensis is the only amphibian capable of surviving several months at almost complete anoxia. We performed 1H NMR metabolomics analysis for the liver and heart of the Siberian frog during normoxia and anoxia. We found that anoxia causes significant energetic stress with much less energy molecules available in the studied organs. This is accompanied by the accumulation of several end products, most significantly lactate, alanine, and succinate, as well as most amino acids.
\end{abstract} frog

Keywords - anoxia, metabolomics, Rana amurensis, Siberian

\section{Motivation and Aim}

Hypoxia is a fatal stress for most terrestrial vertebrates. Only a few species are able to survive prolonged hypoxia, most of them are poikiloterms. The recently discovered ability of the Siberian frog Rana amurensis to survive complete anoxia for three months [1] makes it the only real anoxiatolerant amphibian. Noteworthy, these frogs retain the ability to move and react to stimuli under such conditions.

We performed a metabolomics analysis of the heart an liver of Siberian frogs in normoxia and exposed to anoxia in order to (1) quantify the major metabolites present in the frog tissues; (2) elucidate the energetic pathways used under anoxia and their intensity; (3) estimate the degree of cellular stress during anoxia; and (4) to reveal metabolic adaptations to anoxia.
Methods

A set of six experimental animals was exposed to 1 month of anoxia at near-zero temperature; another group of the same size was kept in normoxia at the same temperature. Hearts and liver of the studied sample were extracted as quickly as possible, frozen in liquid nitrogen and used for metabolite extraction. Identification and quantification of metabolites was performed using $1 \mathrm{H}$ NMR and mass spectrometry analysis.

\section{Results}

Anoxia differed from normoxia in many respects. Glucose, the source of energy for glycolysis, was significantly more abundant during anoxia, as well as end products of glycolysis, lactate and alanine. The Krebs cycle stopped with the accumulation of succinate. Concentrations of most amino acids increased, except for aspartate. Concentrations of AMP and NADH increased severalfold with the concomitant decrease of ATP and NAD+, indicating dramatic decrease in available energetic molecules.

\section{ACKNOWLEDGMENT}

Supported by the RFBR (19-04-00312_a) and by Budget Project of FIZ ICiG SB RAS (0324-2019-0040-C-01).

\section{REFERENCES}

[1] Berman, D. I. et al. (2019). The Siberian wood frog survives for months underwater without oxygen. Scientific reports. 9: 13594 